\title{
MINSKY'S MACROECONOMIC MODELING SOFTWARE PLATFORM OF NONLINEAR DYNAMIC SYSTEMS FROM ECONOMICS
}

\author{
Roxana-Elena Tudoroiu* \\ University of Petrosani, Petrosani, 20 University Street, 332006, Romania \\ *tudelena@mail.com
}

\begin{abstract}
In this paper is presented the flowchart paradigm of one of the most appropriate tools to implement and simulate a nonlinear dynamic system from economics and finance fields, chosen as a case study. More precisely, we talk about the most recent presence on IT market, the so called Minsky macroeconomic modeling software platform. It is similar to Forrester AnyLogic diagrams and MATLAB' SIMULINK software platform widespread in economics and engineering field, respectively. Furthermore, the Minsky software package exhibits realtime simulation features using Godley Tables to generate financial flow equations, in a similar way such the Forrester diagrams from AnyLogic software platform based on the stock-flow concept. The Minsky's macroeconomic software platform is a free open-source computer program, allowing the free download of the most adequate so needed tool for a new approach in economics field. Mostly, it is very useful tool for building and simulation of the large class of dynamic systems, including monetary economic nonlinear models that in majority of cases exhibit naturally a fragile equilibrium characterized by stable or unstable limit cycles. The system dynamics is visually represented by a set of first order differential equations in a multidimensional state-space, and the philosophy behind these differential equations consists of system's structure description with the dynamics arisen from it. Both, simulations and visualization extend the mathematical analysis and provide a good insight about "financial instability hypothesis" and on stable or unstable equilibrium limit cycles similar to those generated by the original Goodwin's model, and its extension known as Minsky's model. If the "financial instability hypothesis" concept, introduced first time by Minsky, is integrated into Goodwin's framework then "converts its stable but cyclical system into a chaotic one, with the possibility of a divergent breakdown-the simulation equivalent of a depression".
\end{abstract}

Keywords: Economy, Lotka-Volterra biological model, Goodwin's and Minsky's models, Minsky's macroeconomic simulation platform, stable and unstable limit cycles, stationary points.

\section{Introduction}

Inspired from the experience accumulated over time by the engineering field in implementation and simulation of thedynamic systems the economics and finance fields have been developed several applications adapted and optimized to the specific economics and financial dynamic models. The dynamics evolution of these models is described through a set of first-order differential equations in a multidimensional state-space. The economics research field is focused on finding a good understanding of economics phenomena such as economic growth, cycles and market analysis to anticipate and control their behavior. Nowadays in economics field are used nonlinear models instead their linearized approximations to capture accurately the entire dynamics of the systems. The use of nonlinear models is justified due to a significant increase of computing power of the PCs desktops, laptops and servers, and to a substantial improvement in simulation tools delivered on the IT market. Also, the desire to understand economic phenomena unable to be captured by their corresponding linearized 
models encourages to develop the nonlinear models. Unfortunately, majority of simulation tools provided by the IT market required to implement the dynamic models are either of high computational complexity to be useful, or depend on local approximations around the operating conditions which fail to adequately capture the nonlinear features of interest.

Let's why in this research paper the readers are informed on the most suitable simulation tools existing on the IT market to implement accurately the nonlinear dynamic systems from economics and finance fields. The proposed Minsky's modeling and simulation platform proves its effectiveness when used on a particular nonlinear Godwin model and its extension to Minsky's financial model considered as a case study. The simulation platform is also computationally simple and easy to be used for nonlinear models implementation. Additionally, it provides a good insight about Minsky's "financial instability hypothesis" on the foundation of Goodwin's limit stable cycle model. Such a financial system will be capable of both generating signals that induce an accelerating desire to invest and of financing that accelerating investment. Minsky's thesis emerges from a simple, structural model of capitalism. The remaining sections are structured as follows. In the second section a historical evolution from differential LotkaVolterra biologic model to Godwin and Minsky model is under consideration. In section 3 is introduced briefly the real time macroeconomic Minsky's modeling platform. The analyzed models and the simulation results on the Minsky's platform are shown in section 4 . Finally, the section 5 concludes by highlighting the main contributions of the paper and states the future work.

\section{Historical Evolution from} Differential Lotka-Volterra Biologic Model to Goodwin and Minsky Models

Lotka-Volterra biologic model is the simplest model of predator-prey interactions. The Lotka-Volterra model describes interactions between two species in an ecosystem, a predator and a prey [1]. This represents the first multi-species model. Since in the model are considered two species, its dynamics is given by the following set of two first order differential equations [1]:

$$
\begin{aligned}
& \frac{d H}{d t}=r H-a H P \\
& \frac{d P}{d t}=b H P-m P
\end{aligned}
$$

First equation describes how the prey population changes, and the second one describes how the predator population also changes. The model was developed independently by Lotka (1925) [3] and Volterra (1926) [4], where the variables $H, P$ represents the density of prey, and the density of predators, respectively.

The model parameters $r, a, b, m$ represents the intrinsic rate of prey population increase, the predation rate coefficient, reproduction rate of predators per 1 prey eaten, and predator mortality rate, respectively. The LotkaVolterra model is one of the earliest predatorprey models to be based on sound mathematical principles [2] that form the basis of many models used today in the analysis of population dynamics. Unfortunately, the model is not very realistic since it does not consider any competition among prey or predators. Thus, it is not surprising that model behavior is unnatural showing no asymptotic stability. However numerous modifications of this model exist which make it more realistic. An extension to a similar predator-prey Lotka-Volterra biologic model is the pathogen model [5], described by a set of three first order differential equations:

$$
\begin{aligned}
& \frac{d S}{d t}=r(S+I)-\gamma P S \\
& \frac{d I}{d t}=\gamma P S-(\alpha+\beta) I \\
& \frac{d P}{d t}=\lambda I-(\mu+\gamma(S+I)) P
\end{aligned}
$$

which describes insect diseases. The host population consist of two portions: susceptible which are healthy organisms, and infected individuals. The model describes changes in density of susceptible $(S)$, infected individuals $(I)$ and pathogens $(P)$. The parameters $\mathrm{r}, \gamma$, $\alpha, \beta, \lambda, \mu$, represent the reproduction rate, the transmission coefficient, the infection rate, the dying rate, emission rate of pathogens, and the natural decay rate of 
pathogens, respectively [5]. This model is capable to generate epidemic cycles, used to study evolutionary strategies of pathogens [5]. Moreover, in [6] is introduced a new extension of prey-predator Volterra model, described the following set of two first order differential equations:

$$
\begin{aligned}
& \frac{d H}{d t}=r H-\lambda H^{2}-a H P \\
& \frac{d P}{d t}=b H P-m P-k P^{2}
\end{aligned}
$$

with all model parameters having positive values. In these equations the fluctuations of $H$ and $P$ are damped, which means that their amplitude diminishes and in time they tend to the equilibrium state [6]. In [7] is introduced a similar two species prey-predator model adapted to a particular macroeconomics nonlinear dynamic Goodwin model that is a simple dynamic model of the cyclical interaction of the growth rates of employment and income distribution.

The set of two first order differential equations (8) and (9) generates the results of the Goodwin model. The first equation captures the profit squeeze relationship and the second one captures the reserve army effect. More precisely, the first equation describes the relationship between the growth of the wage rate $(W)$ and level of unemployment. $(Y)$. This "relies on the reserve army assumption that as unemployment increases the bargaining power of labour is diminished, leading to a fall in the wage rate", as is stated in [7]. This implies also a "relationship between output and the wage share such that higher levels of output will be associated with higher employment and a rising wage share" [7]. The second differential equation describes "the profit squeeze theory of accumulation, in which output growth is constrained by higher real wage rates because investment is assumed to be driven by profits so that as wages rise and profits fall, investment is curtailed and the rate of growth falls", as is stated also in [7]. Therefore, the Goodwin model dynamics is described by the following two differential equations:

$$
\begin{aligned}
& \frac{d Y}{d t}=Y(1-W) \\
& \frac{d W}{d t}=W(-c+r Y)
\end{aligned}
$$

It is worth to mention that in the new version of the Goodwin model it is assumed that "the labour productivity is constant", as is stated in [7]. Thus, the model given by (8) and (9) generates cycles in output around the steady state, while the original Goodwin model is a growth model, revealed also in [7]. At first glance you can see that the couple of equations (8) and (9) is analogous to the Lotka-Volterra (L-V) biological model given in (1) and (2) that describes the dynamic interaction of the populations of two species: a predator and a prey. In the L-V model if the populations of the prey species is higher it leads to growth predator population. Conversely, the rise in the predator population in time causes the prey population to fall. The interaction of these two species generates the cyclical dynamics of the L-V system. Summarizing this section, the concordance between L-V and Goodwin models is given by considering in the Goodwin model, the prey represented by employment levels $(Y)$, and the predator represented by the wage share $(W)$, since a higher wage rate reduces profits and thus causes a fall in investment and growth [7]. By considering the Goodwin model in steady-state, i.e.

$$
\begin{aligned}
& \frac{d Y}{d t}=0 \\
& \frac{d W}{d t}=0
\end{aligned}
$$

two stationary points $(W, Y)$ are obtained as solutions of the following equations:

$$
\begin{gathered}
Y(1-W)=0 \\
W(-c+r Y)=0 \\
\left(W_{e_{1}}, Y_{e_{1}}\right)=(0,0),\left(W_{e_{2}}, Y_{e_{2}}\right)=\left(1, \frac{c}{r}\right)
\end{gathered}
$$

In (7) is shown that first stationary point $\left(W_{e_{1}}, Y_{e_{1}}\right)=(0,0)$ is an unstable saddle-point, and the second one $\left(W_{e_{2}}, Y_{e_{2}}\right)=\left(1, \frac{c}{r}\right)$ is the center of a family of concentric closed orbits, of equation:

$$
\ln (W)-W+c \ln (Y)-r Y=C_{o}
$$

that represents a family of isoclines, each one 
corresponding to a different value of the constant $C_{o}$. The shapes of the orbits (14) are defined by the parameters of the Goodwin model, while the specific orbit generated by the system is determined by the starting values of the variables. The phase diagram for the model is represented in Minsk's simulation platform in the following sections.

\subsection{Minsky's Real Time Macro- Economic Modeling Simulation Platform}

It is a free open-source computer program, a new flowchart paradigm similar to Forrester diagrams based on the stock-flow concept introduced in [10], and an essential simulation tool of the new approach, able to build and simulate engineering dynamic systems, monetary economic models. It is adopted and inspired from engineering modeling and simulation tools but adapted to the specificity of the dynamics models from economics and finance fields. In the most recent Minsky's simulation platform can be implemented and simulated almost all dynamic models, especially those adapted to build monetary models of the economy. However, some work is required to be done in terms of user interface refining, and flexibility to represent the entire economy split in industrial sectors with a good communication between them. Furthermore, is anticipated a promising future in adding new features such as the ability to model international trade and financial flows between different national economies, and also new extensions in importing economic data sets and based on these to derive models parameters by means of nonlinear parameter estimation techniques. The readers interested to apply in their research activity the Minsky's simulation platform can download the program for free via the SourceForge site. In Figure 1, and Figure 2 are shown the Minsky's platform editor with the following components, well described in the platform's manual:

- Mathematical operators such as plus $(+)$, minus (-)

- Constants or parameters whose values are set by the user

- Variables whose values are calculated by the program during a simulation.
- Godley Tables and figure 2 on the platform editor that define both financial accounts and the flows between them. The columns of a Godley table are the stocks, which are computed by integrating over a linear combination of flow variables.

- Integrals representing a variable computed by integrating a function forward in time.

- Groups, which allow components to be grouped into modules that can be used to construct more complex models.

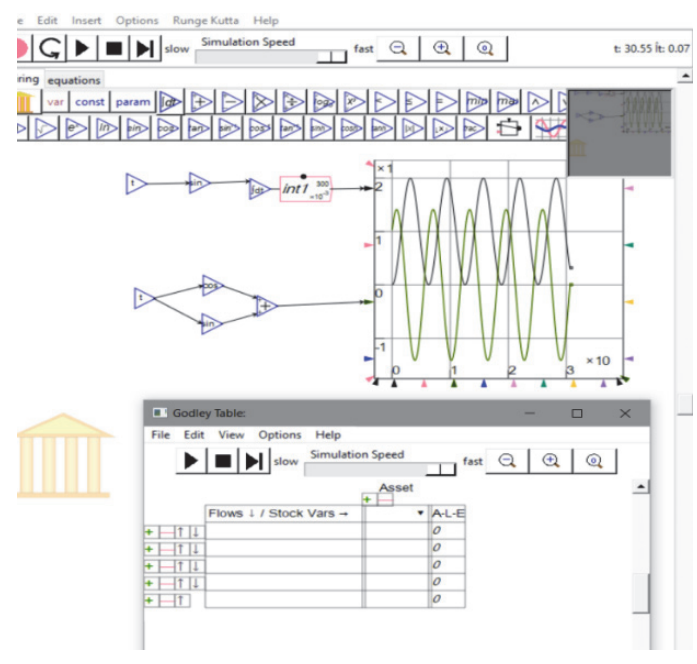

Figure 15. The real time Minsky's simulation platform editor with some of the components (screen snapshot)

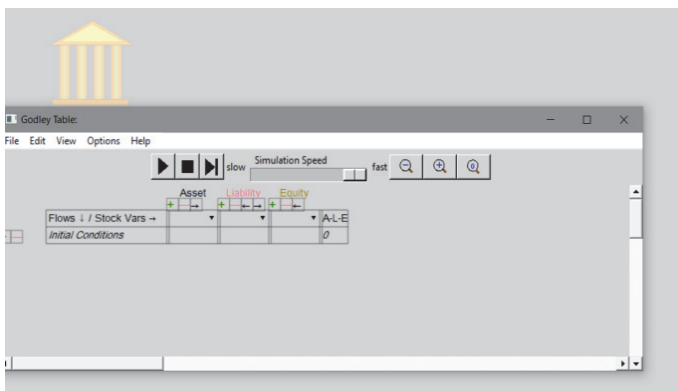

Figure 16. The Godley Table open in Minsky's simulation platform (screen snapshot)

\section{Godwin and Minsky's extension models implementation}

In this section we implement the Godwin and Minsky's extension models. Also the limit 
cycles that occur in the both models are simulated in Minsky's platform.

\subsection{Godwin model and limit cycles implementation}

The simulation results for the following set values of the model parameters and initial conditions for Demand (Y (0) ) and Wage Share (W (0) ):

$$
\begin{aligned}
& c=1.2, r=0.9, \\
& Y(0)=0.5, W(0)=1.25
\end{aligned}
$$

are shown in Figure 3 that reveal the oscillatory behaviour of $\mathrm{Y}$ and $\mathrm{W}$.

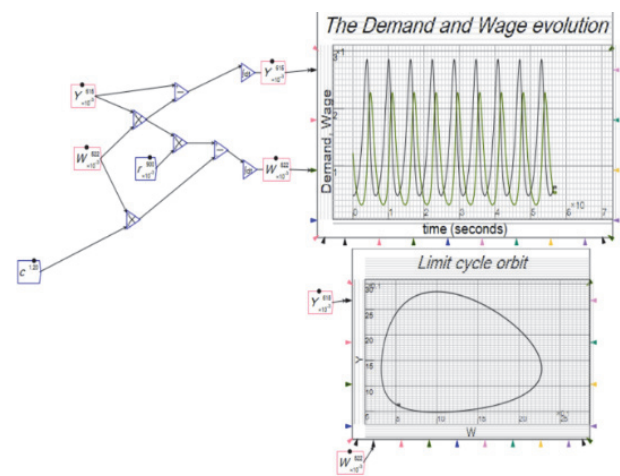

Figure 17. The Godwin model implementation and its limit stable cycle in Minsky's macroeconomic platform

\subsection{The Pseudo-Goodwin cycles in a wage-led Minsky model}

Given the evidence on the strong wealth effects, is interesting to investigate what type of " a finance-driven economy "cycles are generated as response to a wage-led demand regime. The answer is given in [7] by introducing “a highly stylized Minsky's model with a wage-led demand regime and a reserve army distribution function" that proves a great capability feature to analyze the cyclical behavior. The dynamics of the new model is "driven by a Minsky interaction, where higher demand leads to rising financial fragility, i.e. rising debt-to-income ratios, and higher fragility leads to lower growth", as is stated also in [7]. Therefore, higher demand leads to lower unemployment, and positive effects on the wage share are expected. Compared to Goodwin original model given by (8) and (9) described only by two equations, the simplest extension version "wage-led Minsky model" is described by the following three dynamic equations [7], [8]:

$$
\begin{aligned}
& \frac{d F}{d t}=F(-1+p Y) \\
& \frac{d Y}{d t}=Y(1-F+s W) \\
& \frac{d W}{d t}=W(-c+r Y-W)
\end{aligned}
$$

First equation represents the financial fragility $F$, positively related to the demand output $Y$. The second equation is the demand equation with a negative impact of fragility $F$ and a positive one of the wage share $W$. The last equation is "a reserve-army distribution function" that depends positively on output $Y$ and negatively on wage share $W[7,8]$. The "pseudo-Goodwin cycle" is generated only as a side effect as "a reserve-army distribution function" is dragged along by fluctuations in output $Y$ that are driven by financial factors, as is stated in $[7,8]$. The simulation results of Minsky's model implemented and the limit cycles on Minsky platform are displayed in Figure 4 and Figure 5, for the following setting values for model parameters and starting values for fragility, demand and wage share:

$$
\begin{aligned}
& c=1, p=0.95, r=1.6, s=0.1 \\
& F(0)=0.7, Y(0)=0.7, W(0)=0.8
\end{aligned}
$$

The simulation results reveal the instable evolution of fragility, demand and wage share, as well as the stable or unstable Goodwin's limit cycles.

For new settings of model parameters and initial starting values for $\mathrm{F}, \mathrm{Y}, \mathrm{W}$ we get the results from Figure 6, that reveal the same behavior as for the first setting values, only the limit cycles orbits change slightly the appearance, since the radius of the orbits w.r.t. to the new stationay point changes also.

$$
\begin{aligned}
& c=1.5, p=2, r=5, s=0.02 \\
& F(0)=0.75, Y(0)=0.5, W(0)=1
\end{aligned}
$$

The equations, the model parameters values, and the initial conditions for $\mathrm{F}, \mathrm{Y}, \mathrm{W}$, for the new settings can also be visualized by pressing the button Equations from the left top side of the editor above the palette with all operators, constants, parameters and variables, as is shown in Figure 7. 


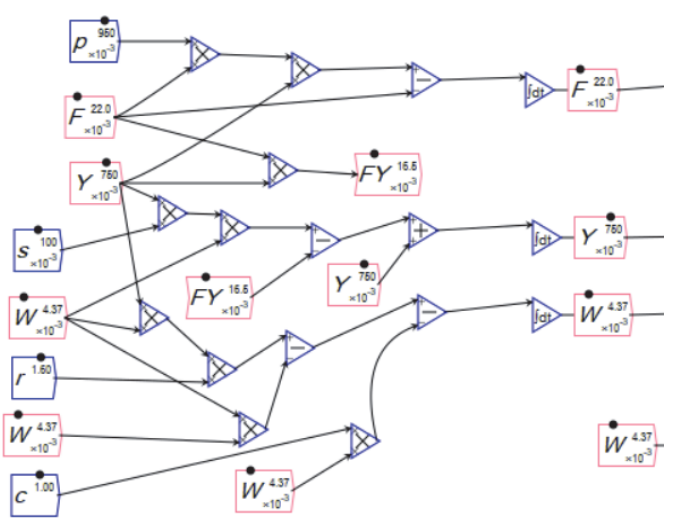

Figure 18. The Minsky's model implemented on Minsky's platform (screen snapshot)
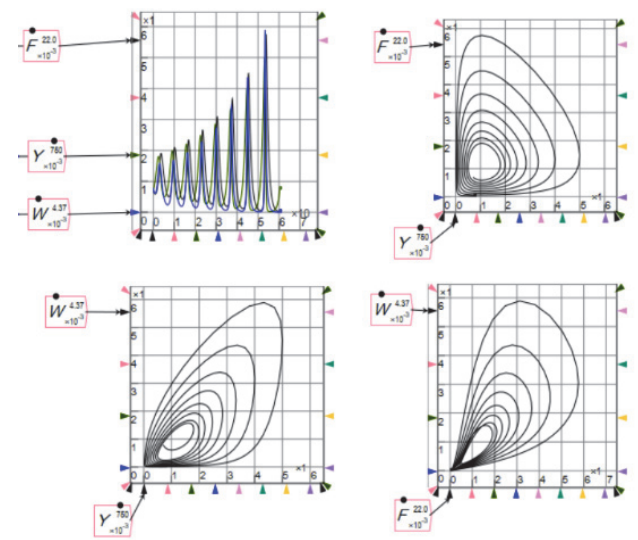

Figure 19. The pseudo Godwin cycles in a wage-led Minsky's model (screen snapshot)

\section{Conclusions}

In this paper is presented the most recent simulation platform adequated to implement and simulate the linear and nonlinear dynamic models encountered in economics and finace applications. Furthermore, the Minsky modeling software platform exhibits real-time simulation features using Godley Tables to generate financial flow equations, in a similar way such as the Forrester diagrams from AnyLogic software platform based also on the stock-flow concept. The readers and the users are well informed now that Minsky's macroeconomic software platform is a free open-source computer program, allowing the free download of the most adequate so needed tool for a new approach in economics field.

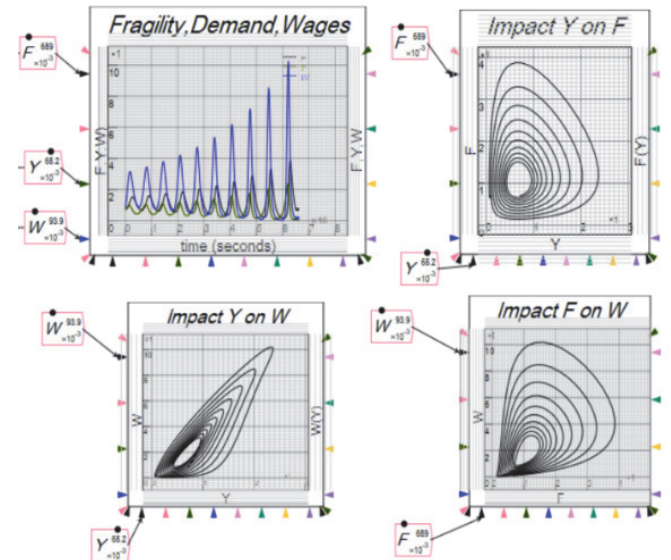

Figure 20. The aspect of limit cycles and F, $\mathrm{Y}, \mathrm{W}$ unstable evolution for the new settings

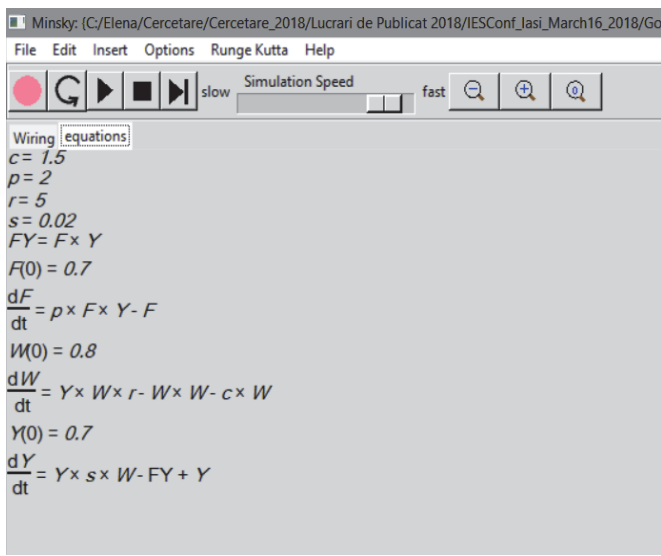

Figure 21. The Minsky's model equations, the setting values of the model parameters and of initial conditions for $\mathrm{F}, \mathrm{Y}$ and $\mathrm{W}$.

This is a very valuable simulation tool found on IT market, user friendly, simple, accurate and all simulations take place in real time. Its effectiveness is proved by an extensive number of simulations and implementations of a particular dynamic models from economics, considered as case study. For future work we are focussed to find many other nonlinear models form economics and finance to implement and simulate, and also to investigate its application to models of high complexity dynamics.

\section{References}

[Internet]. Lotka-Volterra Model. Available on-line on site: //www.ma.utexas.edu/users/davis/375/ 
popecol/lec10/lotka.html. [Accessed March 13, 2018].

[Internet]. Lotka-Volterra Two Species Model.

http:

//www.stolaf.edu/people/mckelvey/envision. dir/lotka-volt.html. [Accessed March 13, 2018].

A.J. Lotka. Elements of physical biology. Baltimore: Williams \& Wilkins Co, (1925) .

V. Volterra. Variazioni e fluttuazioni del numero d'individui in specie animali conviventi. Mem. R. Accad. Naz. dei Lincei. Ser. VI, vol. 2., (1926) .

[Internet] Host-Pathogen Model (Anderson \& May).

https: //www.ma.utexas.edu/users/davis/375/ popecol/lec10/pathogen.html. [Accessed
March 13, 2018].

M-C Anisiu. Lotka, Volterra, and their model.

DIDACTICA MATHEMATICA, Vol. 32 (2014), pp. 9-17.

E. Stockhammer, and J. Michell, "PseudoGoodwin cycles in a Minsky model", Post Keynesian Economics Study Group, Working Paper 1405, May 2014.

E. Stockhammer, "Wage-led versus Profit-led Demand: What have we learned?

A Kalecki-Minsky View", Post Keynesian Economics Study Group, Working Paper 1512, December 2015.

R-E Tudoroiu, "Conceiving and implementing applications using real-time UML", PhD Thesis, Technical University Cluj-Napoca, Romania, 2012. 